\title{
The 2020 Young Innovators of Cellular and Molecular Bioengineering
}

\author{
Michael R. King, ${ }^{1}$ Shelly Peyton, ${ }^{2}$ and Scott I. Simon ${ }^{3}$ \\ ${ }^{1}$ Department of Biomedical Engineering, Vanderbilt University, 5824 Stevenson Center, Nashville, TN 37235, USA; \\ ${ }^{2}$ Department of Chemical Engineering, University of Massachusetts Amherst, N531 Life Sciences Laboratories, 240 Thatcher \\ Rd, Amherst, MA 01003-9364, USA; and ${ }^{3}$ Department of Biomedical Engineering, University of California, Davis, 451 E. \\ Health Sciences Dr., GBSF, Room 3313, Davis, CA 95616, USA
}

(published online 13 October 2020)

We are pleased to present to you this year's 12 Young Innovators of Cellular and Molecular Bioengineering, whose original research is featured in this October issue. It is now the seventh year of the Young Innovators competition, and this year we received many more selfnominations than ever before. All potential authors who hold the rank of Assistant Professor (or equivalent) at the time of nomination are eligible for selection, and while many of the authors are active members of the Biomedical Engineering Society (BMES), membership in BMES is not a requirement for inclusion. The awardees will present the papers in this issue in a special, two-part invited "on-demand" session at the unprecedented Virtual Annual Meeting of BMES. The authors will be available for a live Q\&A session on Friday, October 16, 2020. The articles in this issue represent some of the most innovative and impactful bioengineering studies carried out by junior faculty in our field. This year's Young Innovators are gender balanced and all clearly recognized as rising stars in their field.

Immune engineering attempts to mirror the complexity of a disease process by synthesizing tissue environments with defined architecture and mechanical properties that provide an inner view of how cell function influences health and disease progression. In this context, Teja Guda et al. show how pores in hydroxyapetitie scaffolds with defined size dictate the pattern of vessel sprouting. Abigail Koppes and colleagues on the other hand constructed a model of primary human small intestinal epithelium derived

Address correspondence to Michael R. King, Department of Biomedical Engineering, Vanderbilt University, 5824 Stevenson Center, Nashville, TN 37235, USA. Electronic mail: mike.king@ vanderbilt.edu, speyton@ecs.umass.edu, sisimon@ucdavis.edu from organoids to evaluate the role of acetylcholine receptor activation on inflammatory bowel disease at the level of cytokine induced disruption of the epithelium. To better understand wound healing outcomes, Ashley Brown and her group compared neonatal with adult fibrin scaffolds and discovered distinctions in fibroblast migration thereby revealing how age dependent fibrin porosity and density can be engineered for improved wound healing. Defining a disease process often requires nontraditional approaches to constructing the in vivo environment, to address this Alice Tomei and company reconstructed lymph node mimetic reticula in the pancreas using $3 \mathrm{D}$ collagen sponges to show how to induce tolerance in autoreactive $\mathrm{T}$ cells to avoid $\beta$ cell destruction in type- 1 diabetes. Immune engineering targeted at cancers has found traction as a therapeutic, but modulation of innate immune cells in the process is ill defined. Jamal Lewis and his team report on the immunosuppresive effects of lactic acid secreted by tumors on dentritic cell and macrophage functions, revealing specific pathways that can be targeted to improve antitumor immunity. Amanda Randles led a rigorous study that combined computation and experiment to understand how circulating tumor cells overcome fluid shear stress to metastasize at branch points in the vasculature with particular focus on tumor deformability. Clearly cellular bioengineering applied to solve complex problems and identify the tipping points from health to disease has come of age in these Young, Innovative scientists.

This special issue of Cellular and Molecular Bioengineering also highlights six novel approaches of targeted delivery of proteins, RNAs, and small molecules for a variety of applications. The Chung group at USC developed a targeted delivery system to the kidney via nanoparticles administered through transdermal microneedles. This proved to be a minimally 
invasive platform to deliver biologics specifically to the kidney. The Gallego-Perez lab created a new way to carry oxygen to ischemic areas of tissue via polymerized hemoglobin, which they termed Tissue NanoTransfection. Their method decreased tissue necrosis during ischemia in a mouse skin flap model, with the most striking results found with early intervention. They suggest this method could be a particularly useful early intervention method to combat severe tissue ischemia. The Medina lab published their new work as part of this special issue to repurpose a pathogenspecific antimicrobial peptide, originally designed to kill bacterial Tuberculosis, but retooled as an anticancer peptide. They combined a variety of cell and molecular bioengineering techniques including rational peptide design, advanced microscopy, and flow cytometry to both design and test this approach into typically drug-resistant ovarian cancer cell lines. This could be a valuable approach to find new therapies for patients with drug-resistant tumors.

Out of Vanderbilt University, the Lippman lab developed a SELEX scheme via CRISPR to generate high affinity reagents that selectively bind to membrane proteins in their native conformations. Their proof of concept experiment here targeted the GLUT1 receptor, but this approach will be broadly useful for binding a large diversity of classes of membrane proteins. The Sahay group exploited the properties of naturally occurring lipids to enhance mRNA-based gene transfection. They found a completely unexpected result, that a plant-derived structural lipid (DGTS) significantly improved mRNA transfection in the liver. This work opens the door for discovery of other natural products that could be exploited as nanocarriers for intracellular gene delivery. Finally, the Spangler group engineered an anti-VEGF antagonist protein using concepts from structural biology, molecular engineering, and directed evolution. They expressed this in the choroidal space of rat eyes using nonviral gene delivery, leading to durable expression 1 month after injection. They suggest further library generations of their engineered protein could boost affinity even further, and their approach to targeting VEGF could lead to meaningful treatment options for ocular diseases.

We hope that you will enjoy reading this impressive collection of research articles as much as the reviewers and editors and encourage your eligible colleagues to nominate themselves for next year's CMBE Young Innovator competition. Self-nominations are due by November 9, 2020, with selected authors notified by December 14, 2020, and full manuscripts due by February 15, 2021. Interested researchers who hold a position at the rank of Assistant Professor (or equivalent) are invited to submit a 250-word structured abstract and an NIH-style biosketch to Editor-in-Chief Michael King at mike.king@vanderbilt.edu. You are invited to engage with us via social media on Twitter (www.twitter.com/CMBEjournal) and Facebook (www.facebook.com/CMBEjournal). We hope to see you at the 2020 virtual BMES Annual Meeting, at the 2021 CMBE Conference in Palm Springs, California, and online!

Publisher's Note Springer Nature remains neutral with regard to jurisdictional claims in published maps and institutional affiliations. 